\title{
Some Properties on the Error-Sum Function of Alternating Sylvester Series
}

\author{
Huiping Jing, Luming Shen ${ }^{*}$ \\ Science College of Hunan Agricultural University, Changsha, China \\ Email: Huiping_J@126.com, *lum_s@126.com
}

Received July 11, 2012; revised September 21, 2012; accepted September 29, 2012

\section{ABSTRACT}

The error-sum function of alternating Sylvester series is introduced. Some elementary properties of this function are studied. Also, the hausdorff dimension of the graph of such function is determined.

Keywords: Alternating Sylvester Series; Error-Sum Function; Hausdorff Dimension

\section{Introduction}

For any $x \in(0,1]$, let $d_{1}:=d_{1}(x) \in N$ and $T:=T(x) \in(0,1]$ be defined as

$$
d_{1}(x)=\left[\frac{1}{x}\right],(x):=\frac{1}{d_{1}(x)}-x, T(0):=0 .
$$

where [ ] denote the integer part. And we define the sequence $\left\{d_{n}(x), n \geq 2\right\}$ as follows:

$$
d_{n}(x)=d_{1}\left(T^{n-1}(x)\right) \text {, }
$$

where $T^{n}$ denotes the $n$th iterate of $T\left(T^{0}=I d_{(0,1]}\right)$.

It is well known that from the algorithm (1), all $x \in(0,1]$ can be developped uniquely into an infinite or finite series

$$
\begin{gathered}
x=\sum_{i \geq 1}(-1)^{i-1} \frac{1}{d_{i}(x)}, \\
\text { where }{ }_{i+1}(x) \geq d_{i}(x)\left(d_{i}(x)+1\right) .
\end{gathered}
$$

In the literature [2], (3) is called the Alternating Balkema-Oppenheim expansion of $x$ and denoted by $x=\left[d_{1}(x), \cdots, d_{n}(x), \cdots\right]$ for short. From the algorithm, one can see that $T$ maps irrational element into irrational element, and the series is infinite. While for rational numbers, in fact, we have $x \in(0,1]$ is rational if and only if its sequence of digits $d_{1}(x), \cdots$, is terminate or periodic, see [1-3].

For any $x \in(0,1]$ and $n \geq 1$, define

$$
\frac{p_{n}(x)}{q_{n}(x)}=\sum_{i=1}^{n}(-1)^{i-1} \frac{1}{d_{i}(x)} .
$$

From the algorithm of (1), it is clear that

$$
x=\frac{p_{n}(x)}{q_{n}(x)}+(-1)^{n} T^{n}(x) .
$$

For any $x \in(0,1]$, let $x=\left[d_{1}(x), \cdots, d_{n}(x), \cdots\right]$ be its Alternating Sylvester expansion, then we have $d_{j+1}(x) \geq d_{j}(x)\left(d_{j}(x)+1\right)$ for any $j \geq 1$. On the other hand, any $\left\{d_{j}, j \geq 1\right\}$ of integer sequence satisfying $d_{j+1}(x) \geq d_{j}(x)\left(d_{j}(x)+1\right)$ for all $j \geq 1$ is a Sylvester admissible sequence, that is, there exists a unique $x \in(0,1]$ such that $d_{j}(x)=d_{j}$ for all $j \geq 1$, see [9].

The behaviors of the sequence $d_{n}(x)$ are of interest and the metric and ergodic properties of the sequence $\left\{d_{n}(x), n \geq 1\right\}$ and $T$ have been investigated by a number of authors, see [1-3].

For any $x \in(0,1]$, define

$$
S(x):=\sum_{n=1}^{+\infty}\left(x-\frac{p_{n}(x)}{q_{n}(x)}\right),
$$

and we call $S(x)$ the error-sum function of Alternating Sylvester series. By (4), since $d_{n+1}(x) \geq d_{n}(x)\left(d_{n}(x)+1\right)$ for all $n \geq 1$, then $|S(x)| \leq 1$ and $S(x)$ is well defined. In this paper, we shall discuss some basic nature of $S(x)$, also the Hausdorff dimension of the graph of $S(x)$ is determined.

\section{Some Basic Properties of $S(x)$}

In what follows, we shall often make use of the symbolic space.

For any $n \geq 1$, let

$$
\begin{aligned}
D_{n}= & \left\{\left(\sigma_{1}, \sigma_{2}, \cdots, \sigma_{n}\right) \in N^{n}: \sigma_{k+1} \geq \sigma_{k}\left(\sigma_{k}+1\right)\right. \\
& \text { for all } 1 \leq k \leq n\} .
\end{aligned}
$$

Define

"Corresponding author. 


$$
D=\bigcup_{n=0}^{\infty} D_{n},\left(D_{0}:=\varnothing\right) .
$$

For any $\sigma=\left(\sigma_{1}, \sigma_{2}, \cdots, \sigma_{n}\right) \in D_{n}$, write

$$
\begin{aligned}
& A_{\sigma}=\frac{1}{\sigma_{1}}-\frac{1}{\sigma_{2}}+\cdots+(-1)^{n-1} \frac{1}{\sigma_{n}}, \\
& B_{\sigma}=\frac{1}{\sigma_{1}}-\frac{1}{\sigma_{2}}+\cdots+(-1)^{n-1} \frac{1}{\sigma_{n}+1} .
\end{aligned}
$$

We use $J_{\sigma}$ to denote the following subset of $(0,1]$,

$$
\begin{aligned}
& J_{\sigma}=\left\{x \in(0,1]: d_{1}(x)=\sigma_{1},\right. \\
&\left.d_{2}(x)=\sigma_{2}, \cdots, d_{n}(x)=\sigma_{n}\right\} .
\end{aligned}
$$

From theorem 4.14 of [8], we have $J_{\sigma}=\left(A_{\sigma}, B_{\sigma}\right]$ when $n$ is even, and $J_{\sigma}=\left(B_{\sigma}, A_{\sigma}\right]$ when $n$ is odd. Finally, define

$$
I=\left\{A_{\sigma}, B_{\sigma}, \sigma \in D_{n}, n \geq 1\right\}
$$

Lemma 1. For any $n \geq 1$ and $x \in(0,1]$,

1) $\lim _{x \rightarrow 0^{+}} S(x)=0$

2) $-\frac{17}{30} \leq S(x) \leq 0$;

3) $S(x)=\sum_{i=1}^{n}\left(x-\frac{p_{i}(x)}{q_{i}(x)}\right)+(-1)^{n} S\left(T^{n}(x)\right)$.

Proof. 1) Since $d_{j+1}(x) \geq d_{j}(x)\left(d_{j}(x)+1\right)$ and $d_{1}(x) \geq 1$, so when $n \geq 3$, we can get

$$
d_{n+1} \geq d_{n}^{2}>\cdots>d_{2}^{2^{n-1}},
$$

accordingly

$$
d_{n}>d_{2}^{2^{n-1}} \geq\left(d_{1}^{2}\left(d_{1}+1\right)^{2}\right)^{n-2}
$$

we write $a(x)=d_{1}(x)^{2}\left(d_{1}(x)+1\right)^{2}$, so $d_{n}>a(x)^{n-2}$.

Now $d_{n+1}(x)=\left[\frac{1}{T^{n}(x)}\right]$ implies $\frac{1}{d_{n+1}(x)+1}<T^{n}(x) \leq \frac{1}{d_{n+1}(x)}$, for $0<T^{n}(x) \leq 1$.

Thus

$$
\begin{aligned}
S(x) & =\sum_{n=1}^{+\infty}(-1)^{n} T^{n}(x) \geq \sum_{n=1}^{+\infty}(-1) T^{2 n-1}(x) \\
& \geq \sum_{n=1}^{+\infty}(-1) \frac{1}{d_{2 n}(x)} \geq-\frac{1}{d_{2}(x)}-\sum_{n=2}^{+\infty} \frac{1}{a(x)^{2 n-2}} \\
& \geq-\frac{1}{\sqrt{a(x)}}-\frac{1}{a(x)^{2}-1},
\end{aligned}
$$

let $x \rightarrow 0^{+}$, we have $d_{1}(x) \rightarrow+\infty$ and $a(x) \rightarrow+\infty$, thus

$$
S(x) \rightarrow 0
$$

2) From 1) we know that

$$
d_{n+1} \geq d_{n}^{2}>\cdots>d_{2}^{2^{n-1}}
$$

from the definition of $d_{i}(x)$ we also know that $d_{1} \geq 1$, so $d_{2} \geq d_{1}\left(d_{1}+1\right) \geq 2$,

$$
d_{n+1}>d_{2}^{2^{n-1}} \geq 4^{n-1}
$$

thus

$$
S(x) \geq \sum_{n=1}^{+\infty}(-1) \frac{1}{d_{2 n}(x)} \geq-\frac{1}{2}-\sum_{n=2}^{+\infty} \frac{1}{4^{2 n-2}}=-\frac{17}{30} .
$$

3) Since as $n>m$,

$$
\frac{p_{n}(x)}{q_{n}(x)}-\frac{p_{m}(x)}{q_{m}(x)}=(-1)^{m} \frac{p_{n-m}\left(T^{m}(x)\right)}{q_{n-m}\left(T^{m}(x)\right)} .
$$

Thus

$$
\begin{aligned}
& S(x)=\sum_{i=1}^{\infty}\left(x-\frac{p_{i}(x)}{q_{i}(x)}\right) \\
& =\sum_{i=1}^{n}\left(x-\frac{p_{i}(x)}{q_{i}(x)}\right)+\sum_{i=n+1}^{\infty}\left(x-\frac{p_{n}(x)}{q_{n}(x)}+\frac{p_{n}(x)}{q_{n}(x)}-\frac{p_{i}(x)}{q_{i}(x)}\right) \\
& =\sum_{i=1}^{n}\left(x-\frac{p_{i}(x)}{q_{i}(x)}\right)+\sum_{i=n+1}^{\infty}\left[(-1)^{n} T^{n}(x)-(-1)^{n} \frac{p_{i-n}\left(T^{n}(x)\right)}{q_{i-n}\left(T^{n}(x)\right)}\right] \\
& =\sum_{i=1}^{n}\left(x-\frac{p_{i}(x)}{q_{i}(x)}\right)+(-1)^{n} \sum_{i=1}^{\infty}\left[T^{n}(x)-\frac{p_{i}\left(T^{n}(x)\right)}{q_{i}\left(T^{n}(x)\right)}\right] \\
& =\sum_{i=1}^{n}\left(x-\frac{p_{i}(x)}{q_{i}(x)}\right)+(-1)^{n} S\left(T^{n}(x)\right) .
\end{aligned}
$$

Let

$$
I^{\prime}=I \backslash\{1\} .
$$

Proposition 2. For any $x \in I^{\prime}$, if $x=\left[d_{1}(x), \cdots, d_{2 k+1}(x)\right]$, then $S(x)$ is left continuous but not right continuous. If $x=\left[d_{1}(x), \cdots, d_{2 k}(x)\right]$, then $S(x)$ is right continuous but not left continuous.

Proof. For any $n \geq 1$ and $\sigma \in D_{n}$, write $x_{1}=A_{\sigma}$, $x_{2}=B_{\sigma}$, where $A_{\sigma}, B_{\sigma}$ are given by (6) and (7).

Case I, $n=2 k+1$, then

$$
\begin{aligned}
& x_{1}=\frac{1}{\sigma_{1}}-\frac{1}{\sigma_{2}}+\cdots+\frac{1}{\sigma_{2 k+1}} \\
& x_{2}=\frac{1}{\sigma_{1}}-\frac{1}{\sigma_{2}}+\cdots+\frac{1}{\sigma_{2 k+1}+1}
\end{aligned}
$$

and $J_{\sigma}=\left(B_{\sigma}, A_{\sigma}\right]$. For any $x_{1}^{\prime} \in J_{\sigma}$, since when $\sigma_{2 k+1}=\sigma_{2 k}\left(\sigma_{2 k}+1\right)$, 


$$
\begin{aligned}
& \frac{1}{\sigma_{1}}-\frac{1}{\sigma_{2}}+\cdots-\frac{1}{\sigma_{2 k}}+\frac{1}{\sigma_{2 k+1}} \\
& =\frac{1}{\sigma_{1}}-\frac{1}{\sigma_{2}}+\cdots-\frac{1}{\sigma_{2 k}}+\frac{1}{\sigma_{2 k}\left(\sigma_{2 k}+1\right)} \\
& =\frac{1}{\sigma_{1}}-\frac{1}{\sigma_{2}}+\cdots-\frac{1}{\sigma_{2 k}+1} .
\end{aligned}
$$

This situation is included in Case II, so we can take $\sigma_{2 k+1}>\sigma_{2 k}\left(\sigma_{2 k}+1\right)$ and

$$
x_{1}^{\prime}=x_{1}-\frac{1}{\alpha} \text { for some } \alpha \geq \sigma_{2 k+1}\left(\sigma_{2 k}+1\right) \text {. }
$$

i.e.

$$
\begin{aligned}
x_{1}^{\prime}= & {\left[\sigma_{1}, \cdots, \sigma_{2 k}, \sigma_{2 k+1},[\alpha] \cdots\right] } \\
S\left(x_{1}^{\prime}\right)-S\left(x_{1}\right)= & \sum_{i=1}^{2 k+1}\left(x_{1}^{\prime}-\frac{p_{i}\left(x_{1}^{\prime}\right)}{q_{i}\left(x_{1}^{\prime}\right)}\right)+\left(x_{1}^{\prime}-\frac{p_{2 k+2}\left(x_{1}^{\prime}\right)}{q_{2 k+2}\left(x_{1}^{\prime}\right)}\right) \\
& -\sum_{i=1}^{2 k+1}\left(x_{1}-\frac{p_{i}\left(x_{1}\right)}{q_{i}\left(x_{1}\right)}\right)+S\left(T^{2 k+2}\left(x_{1}^{\prime}\right)\right) \\
= & -\frac{2 k+1}{\alpha}+T^{2 k+2}\left(x_{1}^{\prime}\right)+S\left(T^{2 k+2}\left(x_{1}^{\prime}\right)\right)
\end{aligned}
$$

By (2),

$$
\frac{1}{d_{n+1}(x)+1}<T^{n}(x) \leq \frac{1}{d_{n+1}(x)+1} \text {, for } 0<T^{n}(x) \leq 1,
$$

which implies

$$
\begin{aligned}
T^{n+1}(x) & =\frac{1}{d_{n+1}(x)}-T^{n}(x)<\frac{1}{d_{n+1}(x)}-\frac{1}{d_{n+1}(x)+1} \\
& =\frac{1}{d_{n+1}(x)\left(d_{n+1}(x)+1\right)}
\end{aligned}
$$

and

$$
0<T^{2 k+2}\left(x_{1}^{\prime}\right)<\frac{1}{\alpha(\alpha+1)} .
$$

Let $\alpha \rightarrow+\infty$, we get $T^{2 k+2}\left(x_{1}^{\prime}\right) \rightarrow 0$ and $S\left(T^{2 k+2}\left(x_{1}^{\prime}\right)\right) \rightarrow 0$, thus

$$
\lim _{x_{1}^{\prime} \rightarrow x_{1}^{-}} S\left(x_{1}^{\prime}\right)=S\left(x_{1}\right)
$$

and this implies $S(x)$ is left continuous at $x_{1}$.

Let

$$
\begin{gathered}
x_{1}^{\prime \prime}=x_{1}+\frac{1}{\alpha} \text { for some } \\
\alpha \geq\left(\sigma_{2 k+1}-1\right) \sigma_{2 k+1}\left(\left(\sigma_{2 k+1}-1\right) \sigma_{2 k+1}+1\right), \\
i . e_{1}^{\prime \prime}=\left[\sigma_{1}, \cdots, \sigma_{2 k}, \sigma_{2 k+1}-1,\left(\sigma_{2 k+1}-1\right) \sigma_{2 k+1},[\alpha], \cdots\right],
\end{gathered}
$$

then

$$
\begin{aligned}
& S\left(x_{1}^{\prime \prime}\right)-S\left(x_{1}\right) \\
& =\sum_{i=1}^{2 k}\left(x_{1}^{\prime \prime}-\frac{p_{i}\left(x_{1}^{\prime \prime}\right)}{q_{i}\left(x_{1}^{\prime \prime}\right)}\right)+\left(x_{1}^{\prime \prime}-\frac{p_{2 k+1}\left(x_{1}^{\prime \prime}\right)}{q_{2 k+1}\left(x_{1}^{\prime \prime}\right)}\right) \\
& +\left(x_{1}^{\prime \prime}-\frac{p_{2 k+2}\left(x_{1}^{\prime \prime}\right)}{q_{2 k+2}\left(x_{1}^{\prime \prime}\right)}\right)+\left(x_{1}^{\prime \prime}-\frac{p_{2 k+3}\left(x_{1}^{\prime \prime}\right)}{q_{2 k+3}\left(x_{1}^{\prime \prime}\right)}\right)-S\left(T^{2 k+3}\left(x_{1}^{\prime \prime}\right)\right) \\
& -\left(\sum_{i=1}^{2 k}\left(x_{1}-\frac{p_{i}\left(x_{1}\right)}{q_{i}\left(x_{1}\right)}\right)+\left(x_{1}-\frac{p_{2 k+1}\left(x_{1}\right)}{q_{2 k+1}\left(x_{1}\right)}\right)\right) \\
& =\frac{2 k+2}{\alpha}-\frac{1}{\left(\sigma_{2 k+1}-1\right) \sigma_{2 k+1}}-T^{2 k+3}\left(x_{1}^{\prime \prime}\right)-S\left(T^{2 k+3}\left(x_{1}^{\prime \prime}\right)\right) .
\end{aligned}
$$

Let $\alpha \rightarrow+\infty$, we have

$$
\lim _{x_{1}^{\prime \prime} \rightarrow x_{1}^{+}} S\left(x_{1}^{\prime \prime}\right)=S\left(x_{1}\right)-\frac{1}{\left(\sigma_{2 k+1}-1\right) \sigma_{2 k+1}}
$$

and this implies $S(x)$ is not right continuous at $x_{1}$. For

$$
x_{2}=\frac{1}{\sigma_{1}}-\frac{1}{\sigma_{2}}+\cdots+\frac{1}{\sigma_{2 k+1}+1},
$$

following the same line as above, we have

$$
\lim _{x_{2}^{\prime} \rightarrow x_{2}^{+}} S\left(x_{2}^{\prime}\right)=S\left(x_{2}\right)-\frac{1}{\sigma_{2 k+1}\left(\sigma_{2 k+1}+1\right)} \text {. }
$$

Case II $n=2 k$

Let

$$
\begin{aligned}
& y_{1}=\frac{1}{\sigma_{1}}-\frac{1}{\sigma_{2}}+\cdots-\frac{1}{\sigma_{2 k}} \\
& y_{2}=\frac{1}{\sigma_{1}}-\frac{1}{\sigma_{2}}+\cdots-\frac{1}{\sigma_{2 k}+1}
\end{aligned}
$$

Following the same line as above, we have

$$
\begin{aligned}
& \lim _{y_{1}^{\prime} \rightarrow y_{1}^{-}} S\left(y_{1}^{\prime}\right)=S\left(y_{1}\right)+\frac{1}{\left(\sigma_{2 k}-1\right) \sigma_{2 k}}, \\
& \lim _{y_{2}^{\prime} \rightarrow y_{2}^{-}} S\left(y_{2}^{\prime}\right)=S\left(y_{1}\right)+\frac{1}{\sigma_{2 k}\left(\sigma_{2 k}+1\right)},
\end{aligned}
$$

and $S\left(y_{1}\right), S\left(y_{2}\right)$ is right continuous.

Corollary 3. For any $n \geq 1$ and $\sigma \in D_{n}$, write $\alpha_{1}=\max \left\{A_{\sigma}, B_{\sigma}\right\}, \alpha_{2}=\min \left\{A_{\sigma}, B_{\sigma}\right\}$. Then for any $x \in J_{\sigma}$, if $n=2 k+1$, then

$$
S^{*}\left(\alpha_{2}\right)<S(x) \leq S\left(\alpha_{1}\right)
$$

where $S^{*}\left(\alpha_{2}\right)=S\left(\alpha_{2}\right)-\frac{1}{\sigma_{2 k+1}\left(\sigma_{2 k}+1\right)}$.

From the corollary, for any $\sigma \in D_{n}$

$$
\sup _{x, y \in J_{\sigma}}|S(x)-S(y)|=\frac{n}{\sigma_{n}\left(\sigma_{n}+1\right)}=n \lambda\left(J_{\sigma}\right)
$$


where $\lambda\left(J_{\sigma}\right)$ is the Lebesgue measure of $J_{\sigma}$.

Theorem 4. $S(x)$ is continuous on $(0,1] \backslash I^{\prime}$.

Proof: For any $x \in(0,1] \backslash I^{\prime}$ and $x \neq 1$, let $x=\left(d_{1}(x), \cdots, d_{n}(x), \cdots\right)$ be its Alternating Sylvester expansion. For any $n \geq 1$, write $\sigma^{(n)}=\left(d_{1}(x), \cdots, d_{n}(x)\right)$. By (Corollary 3), for any $y \in J_{\sigma^{(n)}}$, we have

$$
|S(x)-S(y)| \leq(n) \lambda\left(J_{\sigma^{(n)}}\right) \rightarrow 0, \text { as } \rightarrow \infty .
$$

Write $I_{0}=\left\{C_{\sigma}\right\}$, where

$$
C_{\sigma}=\frac{1}{\sigma_{1}}-\frac{1}{\sigma_{2}}+\cdots+\frac{1}{\sigma_{2 k+1}}
$$

Theorem 5. If $0<a<b<1, S(a)<y<S(b)$, then there exists $c \in(a, b) \backslash\left\{I_{0}\right\}$, such that $S(c)=y$.

Proof. Set $g(x)=S(x)-y$, then $g(x)$ has the same continuity as $S(x)$. Write

$$
E=\{x \mid g(x)<0, x \in[a, b]\}, \quad x_{0}=\sup E .
$$

trivially, $a \in E$, then the set is well defined.

If $b=\left[\sigma_{1}, \sigma_{2}, \cdots, \sigma_{2 k+1}\right]$, then by the left continuity of $S(b)$, we have

$$
\lim _{x \rightarrow b^{-}} g(x)=g(b)>0,
$$

As a result, there exists a $\delta_{1}>0$ such that for any $x \in\left(b-\delta_{1}, b\right), g(x)>0$.

If $b=\left[\sigma_{1}, \sigma_{2}, \cdots, \sigma_{2 k}\right]$, since $g(b)$ is not left continuous, then $\exists \delta_{2}>0$ such that for any $x \in\left(b-\delta_{2}, b\right)$, $g(x)>0$, that is $x_{0} \neq b$.

Following the same line as above, we can prove $x_{0}>a$.

Now we shall prove that $g\left(x_{0}\right) \leq 0$. We can choose $x_{n} \in E$ such that $x_{n} \rightarrow x_{0}^{-}$, if $x_{0}=\left[\sigma_{1}, \sigma_{2}, \cdots, \sigma_{2 k+1}\right]$, then

$$
g\left(x_{0}\right)=\lim _{x_{n} \rightarrow x_{0}^{-}} g\left(x_{n}\right) \leq 0,
$$

if $x_{0}=\left[\sigma_{1}, \sigma_{2}, \cdots, \sigma_{2 k}\right]$, then

$$
g\left(x_{0}\right)+\frac{1}{\left(\sigma_{2 k}-1\right) \sigma_{2 k}}=\lim _{x_{n} \rightarrow x_{0}^{-}} g\left(x_{n}\right) \leq 0
$$

In both case $g\left(x_{0}\right) \leq 0$. Following the same line as above, we can prove $g\left(x_{0}\right)=0$, and $x_{0} \neq\left[\sigma_{1}, \sigma_{2}, \cdots, \sigma_{2 k+1}\right]$.

Therefore, there exists $c \in(a, b) \backslash\left\{I_{0}\right\}$, such that $S(c)=y$.

Theorem 6. $\int_{0}^{1} S(x) \mathrm{d} x+\sum_{k=1}^{+\infty} \int_{0}^{\frac{1}{k(k+1)}} S(x) \mathrm{d} x=\frac{9-\pi^{2}}{6}$, and $\int_{0}^{1} S(x) \mathrm{d} x=-0.1250$.

Proof.

$$
\begin{aligned}
& \int_{0}^{1} S(x) \mathrm{d} x=\sum_{d_{1}=1}^{+\infty} \int_{\frac{1}{d_{1}+1}}^{\frac{1}{d_{1}}} S(x) \mathrm{d} x \\
& =\sum_{d_{1}=1}^{+\infty} \frac{\frac{1}{d_{1}}}{\frac{1}{d_{1}+1}}\left(\left(x-\frac{1}{d_{1}}\right)-S(T(x))\right) \mathrm{d} x \\
& =\sum_{d_{1}=1}^{+\infty} \int_{\frac{1}{d_{1}+1}}^{\frac{1}{d_{1}}} x \mathrm{~d} x-\sum_{d_{1}=1}^{+\infty} \int_{\frac{1}{d_{1}+1}}^{\frac{1}{d_{1}}} \frac{1}{d_{1}} \mathrm{~d} x-\sum_{d_{1}=1}^{+\infty} \int_{\frac{1}{d_{1}+1}}^{\frac{1}{d_{1}}} S(T(x)) \mathrm{d} x
\end{aligned}
$$

Let $T x=u=\frac{1}{d_{1}(x)}-x$, then $\mathrm{d} u=-\mathrm{d} x$ thus

$$
\begin{aligned}
\int_{0}^{1} S(x) \mathrm{d} x= & \frac{1}{2} \sum_{d_{1}=1}^{+\infty}\left(\frac{1}{d_{1}^{2}}-\frac{1}{\left(d_{1}+1\right)^{2}}\right) \\
& -\sum_{d_{1}=1}^{+\infty} \frac{1}{d_{1}^{2}}+\sum_{d_{1}=1}^{+\infty} \frac{1}{d_{1}\left(d_{1}+1\right)}-\sum_{d_{1}=1}^{+\infty} \int_{0}^{\frac{1}{d_{1}\left(d_{1}+1\right)}} S(u) \mathrm{d} u
\end{aligned}
$$

thus,

$$
\int_{0}^{1} S(x) \mathrm{d} x+\sum_{k=1}^{+\infty} \int_{0}^{\frac{1}{k(k+1)}} S(x) \mathrm{d} x=\frac{3}{2}-\sum_{d_{1}=1}^{+\infty} \frac{1}{d_{1}^{2}}=\frac{9-\pi^{2}}{6} .
$$

Through the MATLAB program we can get the definite integration

$$
\int_{0}^{1} S(x) \mathrm{d} x=-0.1250
$$

\section{Hausdorff Dimension of Graph for $S(x)$}

Write

$$
\operatorname{Gr}(S)=\{(x, S(x)), x \in(0,1]\} .
$$

Theorem 7. $\operatorname{dim}_{H} \operatorname{Gr}(S)=1$.

Proof. For any $n \geq 1, \quad\left\{J_{\sigma} \times S\left(J_{\sigma}\right), \sigma \in D_{n}\right\}$ is a covering of $\operatorname{Gr}(S)$. From (Cor 3), $J_{\sigma} \times S\left(J_{\sigma}\right)$ can be covered by $n$ squares with side of length $\lambda\left(J_{\sigma}\right)$. For any $\varepsilon>0$,

$$
\begin{aligned}
H^{1+\varepsilon}(G r(S)) & \leq \liminf _{n \rightarrow \infty} \sum_{\sigma \in D_{n}} n(\sqrt{2})^{1+\varepsilon}\left(\lambda\left(J_{\sigma}\right)\right)^{1+\varepsilon} \\
& \leq \liminf _{n \rightarrow \infty} n(\sqrt{2})^{1+\varepsilon} 2^{-n \varepsilon} \sum_{\sigma \in D_{n}} n(\sqrt{2})^{1+\varepsilon} \\
& =\liminf _{n \rightarrow \infty} n(\sqrt{2})^{1+\varepsilon} 2^{-n \varepsilon}=0 .
\end{aligned}
$$

Thus, $\operatorname{dim}_{H} \operatorname{Gr}(S) \leq 1$

Since

$$
|\operatorname{Proj}(x, S(x))-\operatorname{Proj}(y, S(y))| \leq d(x, S(x),(y, S(y)),
$$

then

$$
\begin{aligned}
& \quad 1=\lambda((0,1])=H^{1}(0,1]=H^{1}\left(\operatorname{Proj}\left(G_{r}(S)\right)\right) \leq^{1}\left(G_{r}(S)\right), \\
& \text { so } \operatorname{dim}_{H} G r(S)=1 .
\end{aligned}
$$




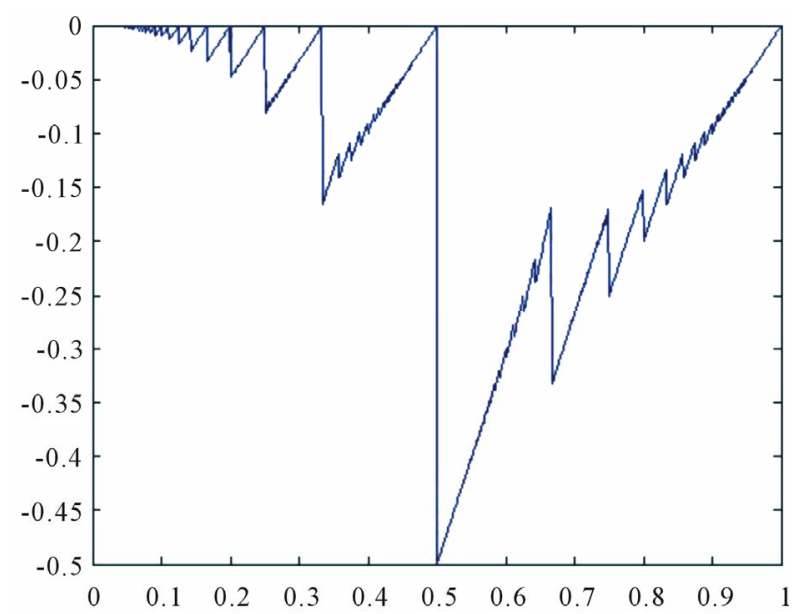

Figure 1. The graph of $S(x)$.

\section{Acknowledgements}

This work is supported by the Hunan Education Department Fund (11C671).

\section{REFERENCES}

[1] S. Kalpazidou, A. Knopfmacher and J. Knopfmacher, "Lüroth-Type Alternating Series Representations for Real Numbers," Acta Arithmetica, Vol. 55, No. 4, 1990, pp. 311-322.

[2] K. H. Indiekofer, A. Knopfmacher and J. Knopfmacher, "Alternating Balkema-Oppenheim Expansions of Real Numbers," Bulletin de la Société Mathématique, Vol.
B44, 1992, pp. 17-28.

[3] S. Kalpazidou, A. Knopfmacher and J. Knopfmacher, "Metric Properties of Alternating Lüroth Series," Potugaliae Mathematica, Vol. 48, No. 3, 1991, pp. 319-325.

[4] J. Barrionuevo, M. Burton-Robert, Dajani-Karma and C. Kraaikamp, "Ergodic Properties of Generalized Lüroth Series," Acta Arithmetica, Vol. 74, No. 4, 1996, pp. 311 327.

[5] K. Dajani and C. Kraaikamp, "On Approximation by Lüroth Series," Journal de Théorie des Nombres de Bordeaux, Vol. 8, No. 2, 1996, pp. 331-346. doi: $10.5802 /$ jtnb. 172

[6] K. J. Falconer, "Fractal Geometry, Mathematical Foundations and Applications," Wiley, Hoboken, 1990.

[7] K. J. Falconer, "Techniques in Fractal Geometry," Wiley, Hoboken, 1997.

[8] J. Galambos, "Reprentations of Real Numbers by Infinite Series," Lecture Notes in Math, Springer, Berlin, 1976.

[9] L. M. Shen and J. Wu, "On the Error-Sum Function of Lüroth Series," Mathematics Analysis and Applications, Vol. 329, No. 2, 2007, pp. 1440-1445.

[10] L. M. Shen, C. Ma and J. H. Zhang, "On the Error-Sum Function of Alternating Lüroth Series," Analysis in Theory and Applications, Vol. 22, No. 3, 2006, pp. 223-232. doi:10.1007/s10496-006-0223-x

[11] T. Sálat and S. Znám, "On the Sums of Prime Powers," Acta Universitatis Palackianae Olomucensis of Mathematica, Vol. 21, 1968, pp. 21-25. 